\title{
Philosophiques
}

\section{La vexante question du normatif}

\section{Alfredo Paternoster}

Volume 30, numéro 2, automne 2003

URI : https://id.erudit.org/iderudit/008650ar

DOI : https://doi.org/10.7202/008650ar

Aller au sommaire du numéro

Éditeur(s)

Société de philosophie du Québec

ISSN

0316-2923 (imprimé)

1492-1391 (numérique)

Découvrir la revue

Citer cet article

Paternoster, A. (2003). La vexante question du normatif. Philosophiques, 30(2), 417-419. https://doi.org/10.7202/008650ar

Ce document est protégé par la loi sur le droit d'auteur. L’utilisation des services d'Érudit (y compris la reproduction) est assujettie à sa politique d'utilisation que vous pouvez consulter en ligne.

https://apropos.erudit.org/fr/usagers/politique-dutilisation/ 


\title{
La vexante question du normatif
}

\author{
ALFREDO PATERNOSTER \\ Université du Piémont Oriental, Vercelli, Italie \\ paternos@eisi.unito.it
}

Dans son Esprit et la nature, Daniel Laurier développe une riche critique du projet philosophique de naturalisation de l'esprit, et notamment de l'intentionnalité, en esquissant une approche alternative, normative, du contenu intentionnel. Dans ce bref commentaire, je ferai deux remarques, l'une concernant la critique des programmes de naturalisation, l'autre concernant la stratégie alternative ébauchée.

Dans le quatrième chapitre, Laurier déroule un argument complexe visant à montrer que si l'intentionnalité est une notion normative, alors le projet de naturalisation est condamné à l'échec. L'argument s'appuie essentiellement sur la considération qu'il y a circularité entre la naturalisation de l'intentionnel et la naturalisation des normes épistémiques. Bien que je ne sois pas convaincu par certains détails de l'argument, je vais l'accepter parce que, entre autres, je suis enclin à croire qu'il n'existe pas de normes naturelles. En revanche, je vais me concentrer sur la prémisse de la thèse conditionnelle.

Pour Laurier, il va sans dire que l'intentionnalité est une notion normative, c'est-à-dire que pour expliquer les phénomènes mentaux (intentionnels), il faut invoquer quelques notions normatives ${ }^{1}$. D'ailleurs, l'idée que c'est d'abord sur la nature normative de l'intentionnalité que se heurtent les tentatives de naturalisation est très commune. Pourtant, je pense que le rôle de la normativité est largement surestimé, tant dans le domaine du mental que dans celui de la signification linguistique. En premier lieu, bien qu'il existe un aspect normatif dans les pratiques de communication linguistique, il n'y a pas de normes véritables. Le seul sens dans lequel la signification linguistique est normative est qu'il y a des usages qui sont (typiquement) tenus comme plus appropriés et des usages (typiquement) tenus comme moins appropriés, en l'absence d'autorités sémantiques ultimes. Ce n'est donc pas exactement une norme qui m'interdit un usage non (ou peu) approprié, et l'on pourrait même soutenir qu'utiliser les mots de manière «standard» est plutôt question de commodité, cela nous convient car cela rend la communication plus efficace ${ }^{2}$. Deuxièmement, si l'on accepte le postulat, tout à fait typique des programmes de naturalisation, que c'est le langage qui dépend de la pensée et non l'inverse, il devient

1. Quelques raisons de croire que l'intentionnalité est normative sont fournies par Daniel Laurier dans un travail à paraître, Pragmatics, Pittsburgh Style. chap. 3 .

2. Voir, par exemple, A. Bilgrami, Belief and Meaning, Oxford, Blackwell, 1992, 
difficile de justifier la normativité de la pensée à partir de sa prétendue nature publique. Il n'est pas évident que la pensée soit publique ${ }^{3}$.

Je concède, toutefois, que l'idée d'après laquelle les états mentaux sont soumis, eux aussi, à des contraintes normatives découle assez naturellement du fait que les états mentaux sont censés avoir de manière essentielle des conditions de vérité. Dans la mesure où dans les programmes de naturalisation (par exemple de Dretske et Fodor), la propriété sémantique cruciale candidate à la réduction est exactement la référence/vérité au sens objectif, propriété conçue à la manière de la sémantique des modèles, des difficultés demeurent.

À la lumière de ces considérations, il me semble que deux options naturalistes s'ouvrent à nous. Dans l'une, qu'on pourrait qualifier de "quasi naturaliste ", les états mentaux sont individualisés de telle manière que l'intentionnalité survient sur des propriétés naturelles (internes) soumises à de modestes facteurs normatifs d'arrière-plan du genre esquissé plus haut. Pourtant, dans ce cas, l'antinaturaliste pourrait légitimement clamer qu'il ne s'agit pas de naturalisation au sens strict, puisqu'elle n'est pas complète. Dans l'autre option, correspondant à ce que Laurier appelle "naturalisme au sens fort ou radical », on renonce bel et bien à la notion de contenu intentionnel. Cela revient à dire que l'intentionnalité n'est qu'une façon de décrire certains aspects des états mentaux, certainement pas une propriété authentique. Ici, l'ami de la normativité sémantique pourrait répondre que, dans la mesure où, interprétés de cette manière, les soidisant programmes de naturalisation de l'intentionnalité sont en réalité des programmes d'élimination de l'intentionnel, cela confirme indirectement la nature non réductible de l'intentionnalité. Je dirais toutefois que, dans la mesure où les phénomènes qui sont typiquement censés être intentionnels sont expliqués en termes naturels, l'intentionnalité est en quelque sorte explained away, et donc que, dans un certain sens, elle est naturalisée.

Le second aspect que j'aimerais discuter dans le livre de Daniel Laurier est son élaboration (bien entendu largement programmatique) d'une approche inférentialiste à la Brandom. Comme Laurier l'affirme, le cadre pragmatiste-inférentialiste de Brandom pourrait être en mesure de donner une explication non circulaire de l'intentionnalité, pourvu que les attitudes pratiques déontiques introduites par Brandom soient considérées des états non conceptuels.

J'ai deux perplexités à cet égard, qui sont peut-être les deux faces d'un seul problème. L'une concerne la plausibilité de concevoir un contenu conceptuel qui (en même temps), d'une part, dépend ou dérive du contenu non conceptuel (en ce sens que n'importe quel système capable d'avoir des états conceptuels doit au préalable avoir des états non conceptuels), et,

3. Voir, par exemple, F. Dretske, "The Nature of Thought», Philosophical Studies, $n^{\circ} 70$ (1993), p. 185-99. 
d'autre part, précède logiquement le contenu non conceptuel, dans le sens qu'il faut des systèmes doués d'états conceptuels pour qu'un système quelconque puisse se trouver dans des états non conceptuels. Même s'il n'y a pas de contradiction véritable dans cette hypothèse ${ }^{4}$, sa défense me paraît une tâche très difficile. L'autre perplexité concerne l'idée même d'après laquelle les attitudes déontiques pratiques seraient des états non conceptuels. Les attitudes déontiques pratiques sont les habiletés de reconnaître quelqu'un en tant qu'autorisé (entitled) ou engagé à produire tel ou tel comportement (linguistique). Or, je dirais que ces attitudes, en tant que formes de know-how, ne sont ni conceptuelles, ni non conceptuelles. Et si l'on prétend tout de même leur donner un contenu, alors je dirai plutôt qu'elles sont conceptuelles, étant donné leur statut normatif. Autrement dit, les attitudes déontiques pratiques me semblent présupposer quelque sorte de compétence sociale, qui entraîne vraisemblablement la possession de certains concepts. Le prototype d'état non conceptuel est plutôt donné par les états perceptuels primaires, ou, mieux, dans une perspective qui vise à caractériser la perception et l'action comme des processus fort intégrés, par les états sensorimoteurs. Quoi qu'il en soit, exploiter la notion de non conceptuel dans le cadre théorique en question me semble viser une difficulté en général, eu égard au fait que (si ma lecture de Brandom est correcte) n'importe quelle attitude n'est intelligible que dans le contexte de la pratique socio-linguistique. Si ces objections ont un fondement, la voie normative pragmatique de l'intentionnalité n'est pas moins difficile que la voie naturaliste non normative.

4. Comme Laurier essaie de le montrer dans la section 6 de Pragmatics, Pittsburgh Style. 